\title{
Assessment of vulnerable and unstable carotid atherosclerotic plaques on endarterectomy specimens
}

\author{
DOINA BUTCOVAN ${ }^{1,2}$, VERONICA MOCANU ${ }^{2}$, DANA BARAN ${ }^{2}$, \\ DIANA CIURESCU ${ }^{1,3}$ and GRIGORE TINICA ${ }^{1,3}$
}

\author{
${ }^{1}$ Department of Cardiovascular Surgery, 'Prof. Dr. George I.M. Georgescu' Institute of Cardiovascular Diseases, \\ Iasi 700503; Departments of ${ }^{2}$ Morpho-Functional Sciences and ${ }^{3}$ Cardiac Surgery, \\ 'Grigore T. Popa' University of Medicine and Pharmacy, Iasi 700115, Romania
}

Received November 30, 2014; Accepted November 25, 2015

DOI: $10.3892 / \mathrm{etm} .2016 .3096$

\begin{abstract}
The types of lesion instability responsible for the majority of acute coronary events frequently include plaque disruption and plaque erosion with superimposed thrombosis. The term 'vulnerable plaque' is used to describe atherosclerotic (ATS) plaques that are particularly prone to rupture and susceptible to thrombus formation, such as the thin-cap fibroatheroma (TCFA). The aim of the present study was to assess the morphological and histological differences between plaques that are unstable and those that are vulnerable to instability. Carotid artery endarterectomy specimens were obtained from 26 patients with carotid artery stenosis, consisting of 20 men and 6 women (age range, 35-80 years). Histological and morphometric methods were used to visualize and characterize the ATS plaques. Among the 26 carotid ATS plaques, $23 \%$ were stable, $23 \%$ were unstable and $54 \%$ were vulnerable. With regard to morphometric characteristics, the following mean values were obtained for the TCFA and unstable plaques, respectively: Fibrous cap thickness, 21.91 and $11.66 \mu \mathrm{M}$; proportion of necrotic core area in the total plaque area, 25.90 and $22.03 \%$; and the proportion of inflammatory area in the total plaque area, 8.41 and $3.04 \%$. No plaque calcification was observed in any of them. Since ATS coronary artery disease is considerably widespread and fatal, it is crucial to further study ATS lesions to obtain an improved understanding of the nature of vulnerable and unstable plaques. The methods used to detect plaque size, necrotic core area and fibrous cap thickness are considered to be particularly useful for identifying vulnerable and unstable plaques.
\end{abstract}

Correspondence to: Professor Veronica Mocanu, Department of Morpho-Functional Sciences, 'Grigore T. Popa' University of Medicine and Pharmacy, 16 Universitatii Street, Iasi 700115, Romania

E-mail: vmocanu@iasi.mednet.ro

Key words: atherosclerosis, calcified nodule, plaque rupture, plaque erosion, vulnerable plaque

\section{Introduction}

Atherosclerotic (ATS) plaques consists of two main components: (i) Soft, lipid-rich atheromatous mass and (ii) hard, collagen-rich sclerotic tissue (1). The sclerotic component, which is fibrous tissue, is the more voluminous component of the plaque which appears to stabilize the plaques and protect against disruption. In contrast, the less voluminous atheromatous component is less stable as the soft atheromatous gruel destabilizes the plaque, thus leaving it vulnerable to rupture. Upon plaque rupture, the highly thrombogenic gruel is exposed to the blood, leading to thrombosis, which is a characteristic feature of unstable plaques (1).

The majority of disrupted plaques have been shown to have a large necrotic core with a thin overlying fibrous cap, with substantial inflammation and limited calcification (2). It has been hypothesized that they do not cause critical narrowing of the carotid lumen, due to outward (expansive or positive) remodeling of the arterial segment (3). In addition, it has been suggested that lesions with similar histomorphological characteristics, but intact fibrous caps, have a high risk of rupture (2).

The aim of the present study was to characterize a number of carotid ATS plaques, which were obtained by endarterectomy, for the purpose of determining the importance of various pathological characteristics, including fibrous cap thickness and plaque, necrotic core, macrophage and calcification areas. The study included stable plaques [SP; including fibroatheromas (FAs) and fibrocalcified plaques (FCs)], vulnerable plaques [VP; including thin-cap fibroatheromas (TCFAs)], and unstable plaques [USP; featuring plaque rupture (PR), plaque erosion (PE) and calcified nodules $(\mathrm{CNs})]$, as previously defined by pathologists (4).

The present study elucidated the associations between plaque characteristics and the risk factors for the development of CVD including age, smoking, arterial hypertension, hyperlipidemia and diabetes mellitus. We hypothesized that the present study may contribute to the improved understanding of acute coronary events and underlying plaque composition and its association with coronary risk factors, in order to improve the timely identification of vulnerable plaques. 


\section{Materials and methods}

Patients. A total of 26 patients that underwent carotid artery endarterectomy to treat high-grade internal carotid artery stenosis at the 'Prof. Dr. George I.M. Georgescu' Institute of Cardiovascular Diseases (Iasi, Romania) between January and December 2013 participated in the present study. Excised carotid ATS plaques were obtained from 20 male and 6 female patients, aged 35-80 years, who presented with transient ischemic attack symptoms upon diagnostic consultation. Ethical approval for the present study was granted by the Institutional Board at Prof. Dr. George I.M. Georgescu' Institute of Cardiovascular Diseases. Written informed consent was obtained from the patients.

Histological examination. Carotid endarterectomy was performed under anesthesia using fentanyl (5-15 mcg/kg; Gedeon Richter Plc., Budapest, Hungary), midazolam (0.05-0.1 mg/kg; Hofman-La Roche Ltd., Basel, Switzerland), and rocuronium $(0.6 \mathrm{mg} / \mathrm{kg}$; Fresenius Kabi Austria GmbH, Graz, Austria) for induction and maintained with isoflurane (Rompharm Company, Otopeni, Romania). The entire intimal carotid plaques (length, $\sim 1 \mathrm{~cm}$ ) were removed from the carotid arteries. Excessively fragmented plaques ( $>2$ pieces) were not subjected to further analysis. The carotid artery atherosclerotic plaques were subsequently sectioned at 3-4-mm intervals and processed for histological examination. All sections were stained with hematoxylin and eosin, elastic Van Gieson, Masson's trichrome and movat pentachrome (all Bio Optica Milano SpA, Milan, Italy). Hematoxylin stains the nuclei and calcified material in cells blue, whereas eosin stains eosinophilic structures in various shades of red. Elastic Van Gieson is a histological stain for elastic and collagen fibers and Masson's trichrome and movat pentachrome stain muscle fibers, collagen and nuclei. Masson's trichome stain is used to detect connective tissue (green) and muscle tissue (red) characterized by fibrotic and degenerative changes; whereas movat pentachrome stain differentiates between the various ages and types of collagen and connective tissue matrix in the ATS plaques. Microscopic sections were analyzed with the observer blinded to the clinical status of the patients whose plaques were being examined.

Classification. ATS plaques were classified as either VP, SP or USP, as previously described (5). Lesions displaying a thin fibrous cap with infiltration of macrophages and a large necrotic core containing numerous cholesterol clefts were defined TCFA, a type of VP. In carotid TCFAs, the area of the necrotic core is $\leq 3 \mathrm{~mm}^{2}$, and the cap thickness of a vulnerable lesion is $\leq 165 \mu \mathrm{m}$. With regard to SPs, those with a large lipid-necrotic core containing extracellular lipid, cholesterol crystals and necrotic debris covered by a thick fibrous cap were considered to be FAs, whereas plaques with a small or absent lipid-laden necrotic core and a thick fibrous cap overlying extensive accumulations of calcium in the intima close to the media were considered FC plaques. In USP thrombotic plaques, thrombi occur as a consequence of one of the three following events: PR, PE or, less frequently, CNs. PR was defined as an area of fibrous cap disruption in which the overlying thrombus was in continuity with the underlying necrotic core. PE was
Table I. Carotid atherosclerotic plaques.

\begin{tabular}{lll}
\hline & \multicolumn{2}{c}{ Patients $(\mathrm{n}=26)$} \\
\cline { 2 - 3 } Plaque type & $\mathrm{n}$ & $\%$ \\
\hline Stable & 6 & 23 \\
Fibroatheroma & 4 & \\
Fibro-calcified & 2 & 54 \\
Vulnerable & 14 & \\
Thin-cap fibroatheroma & 14 & \\
Unstable & 6 & \\
Plaque erosion & 2 & \\
Plaque rupture & 2 & \\
Calcified nodules & 2 & \\
\hline
\end{tabular}

identified when a thrombus covers a fibrous cap with no defect. Typically, the endothelium is absent from the erosion site. CNs are lesions with fibrous cap disruption and thrombi associated with dense nodules exhibiting calcification.

Histomorphometric analysis. Histological assessment was performed by an experienced pathologist using an optical microscope (CX41; Olympus Corporation, Tokyo, Japan). The measurements were visualized using color image analysis software (QuickPHOTO MICRO 3.0, PROMICRA, S.r.o., Prague, Czech Republic). Quantitative morphometry included measurement of the plaque, lipid necrotic core, inflammatory and calcified areas and fibrous cap thickness.

Risk factor assessment. The following potential risk factors (RFs) for atherosclerosis were also assessed: Age, gender, diabetes mellitus (DM), arterial hypertension, history of cigarette smoking, cerebrovascular diseases and hyperlipidemia. All RF data was obtained from the patients' files.

Statistical analysis. The association among the pathological characteristics of the three defined plaque types, as well as among plaque types and ATS RFs were evaluated. Data are expressed as mean values and percentages, calculated using Excel software (Microsoft Corporation, Redmond, WA, USA).

\section{Results}

Plaque types. A total of 26 carotid ATS plaques were histologically analyzed and classified as follows (Table I): i) 6 cases of SP (23\%), including 4 fibroatheroma (Fig. 1A) and 2 fibrocalcified plaques (Fig. 1B); ii) 14 cases of VP (54\%), indicated by TCFA (Fig. 1C); and iii) 6 USPs (23\%), including 2 cases each of PE (Fig. 2A), PR (Fig. 2B) and CNs (Fig. 2C).

Histomorphometric analysis. Morphometric analysis was performed to evaluate the fibrous cap thickness and necrotic core size, in addition to the inflammatory and calcified plaque areas of the ATS plaques (Table II).

USPs exhibited the smallest fibrous cap thickness and a large lipid core area in the total plaque area, and exhibited 
Table II. Carotid atherosclerotic plaque measurements.

\begin{tabular}{llccc}
\hline Plaque type & Fibrous cap, $\mu \mathrm{m}$ & Necrotic core, $\%$ & Inflammatory infiltrate, $\%$ & Calcification, $\%$ \\
\hline FA & $351(173-654)$ & $56.99(32.24-87.19)$ & Minimum & - \\
FC & $270(170-371)$ & $46.86(37-56.72)$ & - & $6.23(8.11-4.36)$ \\
TCFA & $21.91(5-44)$ & $25.90(3.04-60.22)$ & $8.41(0-25.87)$ & - \\
PR & $11.66(6-20)$ & $22.03(2.64-32.50)$ & $3.04(2.64-32.50)$ & - \\
PE & $13.13(10-16)$ & $70.29(51.97-87.74)$ & $6.69(5-8.35)$ & $17.85(14.11-21.46)$ \\
CN & $20.33(11-35)$ & - & Minimum & 136.599 \\
\hline
\end{tabular}

FA, fibroatheroma; FC, fibro-calcified plaque; TCFA, thin-cap fibroatheroma; PR, plaque rupture; PE, plaque erosion; CN, calcified nodule.

A

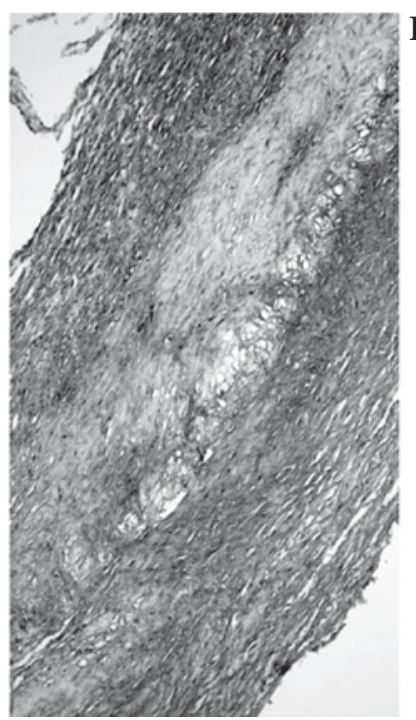

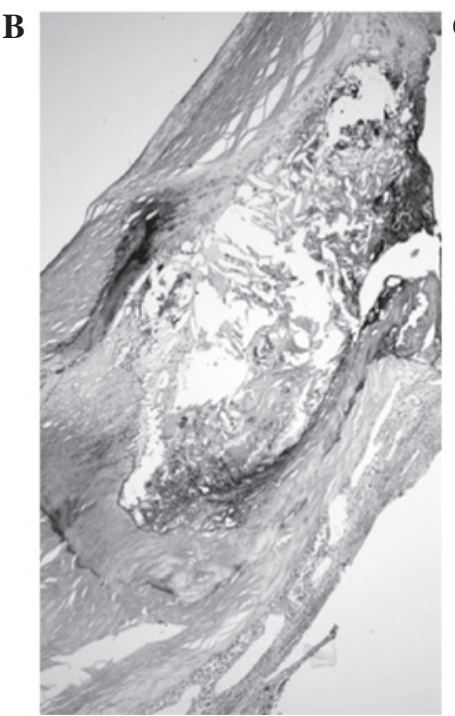

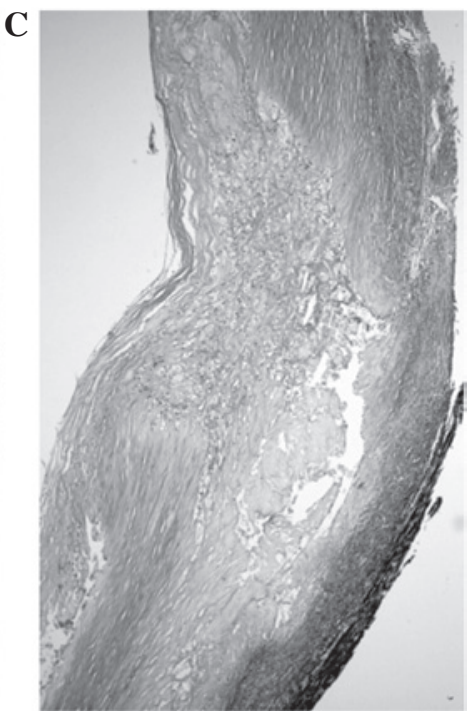

Figure 1. (A) Fibroatheromatous plaque (stain, Masson's trichrome; magnification, x10); (B) Fibro-calcified plaque (stain, elastic Van Gieson; magnification, x10); (C) Thin-cap fibroatheroma (stain, elastic Van Gieson; magnification, x10).

A

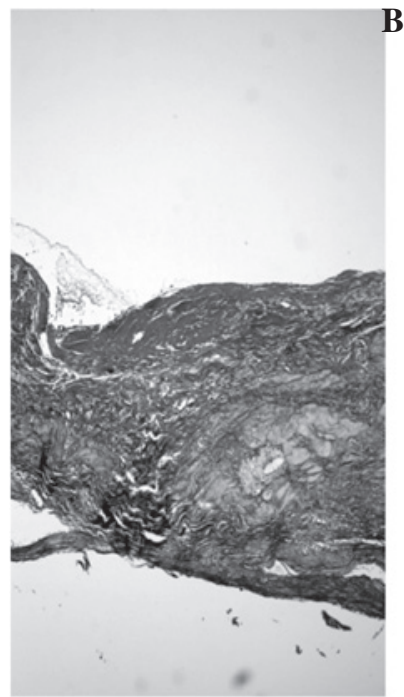

B

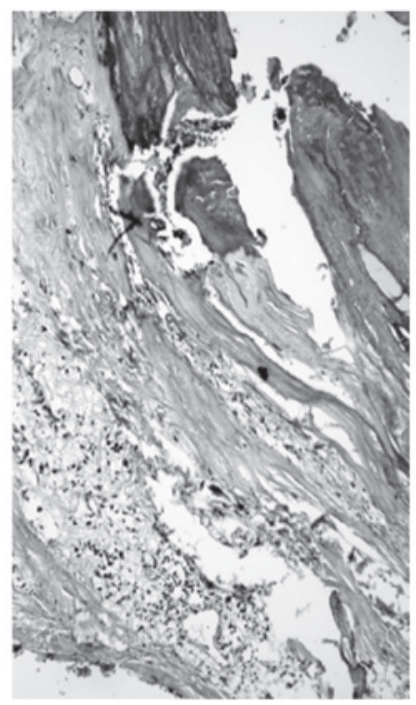

C

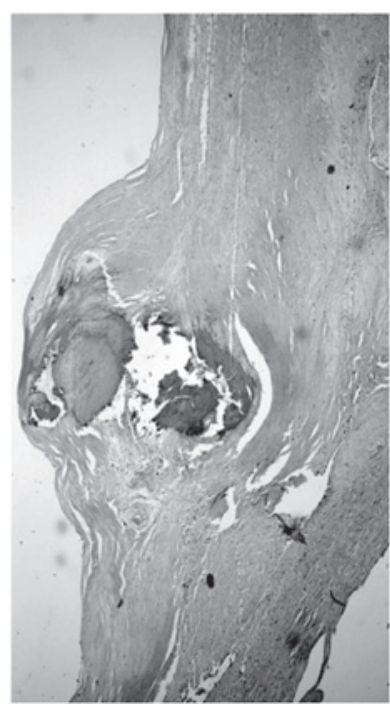

Figure 2. (A) Erosion plaque (stain, Masson's trichrome; magnification, x10); (B) Ruptured plaque (stain, movat pentachrome; magnification, x10); (C) Calcified nodule (stain, hematoxylin and eosin; magnification, x10).

inflammatory infiltrate. VPs were found to have a fibrous cap thickness of $<164 \mu \mathrm{m}$ and lipid core areas of varying sizes, and showed considerable inflammatory infiltrate. Plaque instability was found to be highly associated with fibrous cap thickness, lipid core area and plaque size. The incidence of the various RFs is summarized in Table III. 
Table III. Incidence rates of risk factors in relation to ATS lesions.

\begin{tabular}{lcccccc}
\hline & \multicolumn{5}{c}{ Risk factors (n) } \\
\cline { 2 - 7 } ATS lesion & Age $>50$ years & Smoking & AHT & HL & DM & CVD \\
\hline SP & 2 & 1 & 5 & 2 & 1 & 0 \\
VP & 10 & 4 & 14 & 8 & 4 & 10 \\
USP & 5 & 2 & 6 & 3 & 4 & 5 \\
Total patients (\%) & $17(65.38)$ & $7(26.92)$ & $25(96.15)$ & $13(50.00)$ & $9(34.61)$ & $15(57.69)$ \\
\hline
\end{tabular}

ATS, atherosclerotic; AHT, arterial hypertension; HL, hyperlipidemia; DM, diabetes mellitus; CVD, cardiovascular diseases; SP, stable plaques; VP, vulnerable plaques; USP, unstable plaques.

Table IV. Incidence of the cumulative cardiovascular risk factors in each patient.

\begin{tabular}{lcccccc}
\hline & \multicolumn{5}{c}{ Cardiovascular risk factors (n) } \\
\cline { 2 - 6 } ATS lesion & 6 & 5 & 4 & 3 & 2 & 1 \\
\hline SP & 0 & 0 & 2 & 0 & 0 & 0 \\
VP & 3 & 4 & 10 & 1 & 0 & 0 \\
USP & 2 & 1 & 3 & 0 & 0 & 0 \\
Total patients (\%) & $5(19.23)$ & $5(19.23)$ & $15(57.69)$ & $1(3.84)$ & $0(0.00)$ & $0(0.00)$ \\
\hline
\end{tabular}

ATS, atherosclerotic; SP, stable plaques; VP, vulnerable plaques; USP, unstable plaques.

The age of the patients, as well as the prevalence of the RFs for ATS differed between the SP and USP groups (data not shown). Arterial hypertension was found to be coexistent with the development of ATS plaques in $96.15 \%$ of all cases. Furthermore, age appeared to be an associated factor in ATS development. Hyperlipidemia was detected in $50 \%$ of all cases. DM was present in $34.61 \%$ of cases and was one of the investigated RFs involved in ATS progression in the vessels. Smoking was involved in $26.92 \%$ of all cases, suggesting that it is an important risk factor in early and late thrombosis. Involvement of other peripheral vessels in ATS process was identified in $57.69 \%$ of all cases.

Risk factors. The age of the patients and the prevalence of the RFs for ATS in the SP group differed from the USP group: In order of decreasing frequency, the RFs were higher in VPs compared with the USPs and SPs (data not shown). Table IV shows the cumulative RFs in each patient.

Taking into consideration the number of the RFs involved in the pathogenesis of ATS in each patient, the following frequencies were observed (Table IV): One patient (3.84\%) had three RFs, 15 patients (57.69\%) had 4 RFs, 5 patients (19.23\%) had 5 RFs, and 5 patients $(19.23 \%$ ) had 6 RFs. No patient was found to have 1 or 2 RFs. The most frequent cumulative factors were identified in patients with VPs, followed by patients with USPs.

\section{Discussion}

Atherosclerosis is a major cause of morbidity and mortality worldwide, and despite the advances in the understanding of its pathogenesis, the factors that determine atheromatous plaque instability remain largely unknown. The prediction of plaque vulnerability to rupture and subsequent thrombosis would be useful in the development of diagnostic and therapeutic approaches (2). By correlating histomorphological examinations and imaging results, the present study aimed to develop an improved criteria for the diagnostic processing of ATS plaques.

Cap thickness has been identified as a crucial characteristic for the distinction between TCFAs and FAs, as TCFAs are known to have a thinner cap (4). However, only 1 case of plaque rupture $(2.66 \%)$ and 3 cases of TCFAs $(12.5 \%)$ had a cap thickness $>15 \mu \mathrm{m}$. Moreno (2), suggested that monitoring cap thickness may be an option for predicting future behavior of an ATS lesion (6).

Further separation of plaque ruptures and TCFAs from FAs is indicated by the simultaneous presence of plaque inflammation and necrotic core area (3). Regarding FAs, definitory issues were cap thickness $\geq 165 \mu \mathrm{m}$ and necrotic core area $\geq 3.5 \mathrm{~mm}^{2}(4)$.

Excluding fibrous cap thickness from the analysis, plaque inflammation allowed for the separation of plaque rupture and TCFAs from FAs with a proportion of $\geq 3$ times greater in VPs and RPs compared with FAs (6). Hirayama et al (7) found a discriminatory level of inflammation consisting in $>0.2 \mathrm{~mm}^{2}$ macrophage area/high-power microscopic field.

In a previous study, the mean area of macrophages per plaque area, infiltrating the region of the fibrous cap and the shoulder was significantly greater in the non-calcified plaque sections compared with the calcified sections (7). Although the 
present study had certain limitations, including the low sample size and lack of statistical analysis, the results indicated that a larger macrophage-rich area is present in the fibrous cap and the shoulder region of noncalcified plaques. Furthermore, noncalcified carotid plaques commonly exhibit a higher degree of fibrous cap inflammation, a key process in fibrous cap disruption.

Calcification in ATS lesions is relatively common and has been implicated as a risk factor for increased cardiovascular morbidity and mortality $(8,9)$, while Bayturan et al (10) and Hashimoto et al (11) demonstrated that carotid ATS plaque calcification is a structural marker for carotid plaque stability.

In the present study, 14 plaques were defined as TCFAs. These plaques were not ruptured but were considered of high risk; thus, detection of such plaques is the key aim of diagnosis.

PRs and fissures usually occur in the fibrous cap and shoulder regions. Rupture of the ATS plaques is responsible for the majority of acute coronary events, and such lesions have been shown to exhibit distinct histopathological features $(12,13)$. The present results indicated that fibrous cap inflammation and susceptibility to disruption are more likely to occur in noncalcified compared with calcified plaques. We speculate that the quantitative assessment of carotid plaque calcification using imaging modalities may help identify patients with asymptomatic vulnerable carotid plaques who are at risk of cerebrovascular ischemic events and would benefit from carotid interventions.

Intimal thickness and lipid core area have been associated with RFs for coronary ATS disease (14), and may be valid markers of early carotid atherosclerosis from pathology. In the present study, the prevalence of hypertension, hyperlipidemia, and current history of smoking was higher in symptomatic than compared with asymptomatic cases (data not shown). Therefore, the identification of RFs for carotid atherosclerosis among patients with coronary heart disease may provide an evidential basis for prevention.

Currently, among the most promising fields in the study of atherosclerosis is the development of imaging techniques that facilitate gross, microscopic and molecular characterization of in vivo ATS plaques (2). Modern visualization techniques are able to demonstrate the presence of inflammation, macrophage infiltration, angiogenesis, apoptosis, and other cellular and molecular features of plaques that may be involved in plaque destabilization and subsequent clinical events even in living patients (15). In addition, a recent computed tomography coronary angiography study by Versteylen (3) demonstrated that large plaques with calcified micronodules are the most prone to rupture. Such imaging techniques may facilitate the more specific characterization of ATS plaques and the identification of characteristics that are associated with and directly responsible for plaque rupture. All these approaches; however, should only complement histopathological investigations, which more specifically confirm the identity of lesions.

Atheromatous plaques may become unstable due to increases in size, increased intra- and extracellular lipid accumulation, as well as intraplaque hemorrhage (16). Based on these results, diagnostic modalities that detect plaque size, and hemorrhage, and/or lipid content are most likely to be useful in the prediction of unstable plaques. Furthermore, atheromatous plaques may become vulnerable due to fibrous cap thinning, which is associated with cap inflammation, a key process in fibrous cap disruption (17). Therefore, diagnostic methods that detect plaque size and hemorrhage, lipid content and the thinnest fibrous cap can prove considerably useful in determining USPs and VPs.

The most common RFs that were found to be associated with stable, vulnerable and unstable ATS plaques were age and hypertension. Hypertension in atherosclerosis indicates disease progression and may become life-threatening $(1,16)$; however, associations between specific risk factors and the composition of plaque are yet to be elucidated.

\section{References}

1. Falk E, Shah PK and Fuster V: Coronary plaque disruption. Circulation 92: 657-671, 1995.

2. Moreno PR: Vulnerable plaque: Definition, diagnosis and treatment. Cardiol Clin 28: 1-30, 2010

3. Versteylen MO: Additive value of semi-automated quantification of coronary artery disease using cardiac computed tomographic angiography to predict for future acute coronary syndrome. J Am Coll Cardiol 61: 2296-2305, 2013.

4. Furie KL, Kasner SE, Adams RJ, Albers GW, Bush RL, Fagan SC, Halperin JL, Johnston SC, Katzan I, Kernan WN, et al; American Heart Association Stroke Council, Council on Cardiovascular Nursing, Council on Clinical Cardiology, and Interdisciplinary Council on Quality of Care and Outcomes Research: Guidelines for the prevention of stroke in patients with stroke or transient ischemic attack: A guideline for healthcare professionals from the american heart association/american stroke association. Stroke 42: 227-276, 2011.

5. Virmani R, Kolodgie FD, Burke AP, Farb A and Schwartz SM: Comprehensive Morphological Classification of Atherosclerotic Lesions. Arterioscler Thromb Vasc Biol 20: 1262-1275, 2000.

6. Fishbein MC: The vulnerable and unstable atherosclerotic plaque. Cardiovasc Pathol 19: 6-11, 2010.

7. Hirayama A, Saito S, Ueda Y, Takayama T, Honye J, Komatsu S, Yamaguchi O, Li Y, Yajima J, Nanto S, et al: Qualitative and quantitative changes in coronary plaque associated with atorvastatin therapy. Circ J 73: 718-725, 2009.

8. Brajovic MD, Marković N, Loncar G, Sekularac N, Kordić D, Despotović N, Erceg P, Donfrid B, Stefanović Z, Bajcetić M, et al: The influence of various morphologic and hemodynamic carotid plaque characteristics on neurological events onset and deaths. ScientificWorldJournal 9: 509-521, 2009

9. Kim SH, Hong MK, Park DW, Lee SW, Kim YH, Lee CW, Kim JJ, Park SW and Park SJ: Impact of plaque characteristics analyzed by intravascular ultrasound on long-term clinical outcomes. Am J Cardiol 103: 1221-1226, 2009.

10. Bayturan O, Tuzcu EM, Nicholls SJ, Balog C, Lavoie A, Uno K, Crowe TD, Magyar WA, Wolski K, Kapadia S, et al: Attenuated plaque at nonculprit lesions in patients enrolled in intravascular ultrasound atherosclerosis progression trials. JACC Cardiovase Interv 2: 672-678, 2009.

11. Hashimoto H, Tagaya M, Niki $\mathrm{H}$ and Etani $\mathrm{H}$ : Computer-assisted analysis of heterogeneity on B-mode imaging predicts instability of asymptomatic carotid plaque. Cerebrovasc Dis 28: 357-364, 2009.

12. Mortaz Hejri S, Mostafazadeh D and Sahraian M: Carotid endarterectomy for carotid stenosis in patients selected for coronary artery bypass graft surgery. Cochrane Database Syst Rev: CD006074, 2009.

13. Finn AV, Kramer MC, Vorpahl M, Kolodgie FD and Virmani R: Pharmacotherapy of coronary atherosclerosis. Expert Opin Pharmacother 10: 1587-1603, 2009.

14. WRITING GROUP MEMBERS; Lloyd-Jones D, Adams RJ, Brown TM, Carnethon M, Dai S, De Simone G, Ferguson TB, Ford E, Furie K, Gillespie C, et al; American Heart Association Statistics Committee and Stroke Statistics Subcommittee: Heart disease and stroke statistics - 2010 update: A report from the American Heart Association. Circulation 121: e46-e215, 2010.

15. Motoyama $S$, Sarai $M$, Harigaya $H$, Anno $H$, Inoue $K$, Hara $T$, Naruse H, Ishii J, Hishida H, Wong ND, et al: Computed tomographic angiography characteristics of atherosclerotic plaques subsequently resulting in acute coronary syndrome. J Am Coll Cardiol 54: 49-57, 2009.

16. Van der Wal AC and Becker AE: Atherosclerotic plaque rupture - pathologic basis of plaque stability and instability. Cardiovasc Res 41: 334-344, 1999.

17. Kolodgie FD, Virmani R, Burke AP, Farb A, Weber DK, Kutys R, Finn AV and Gold HK: Pathologic assessment of the vulnerable human coronary plaque. Heart 90: 1385-1391, 2004. 